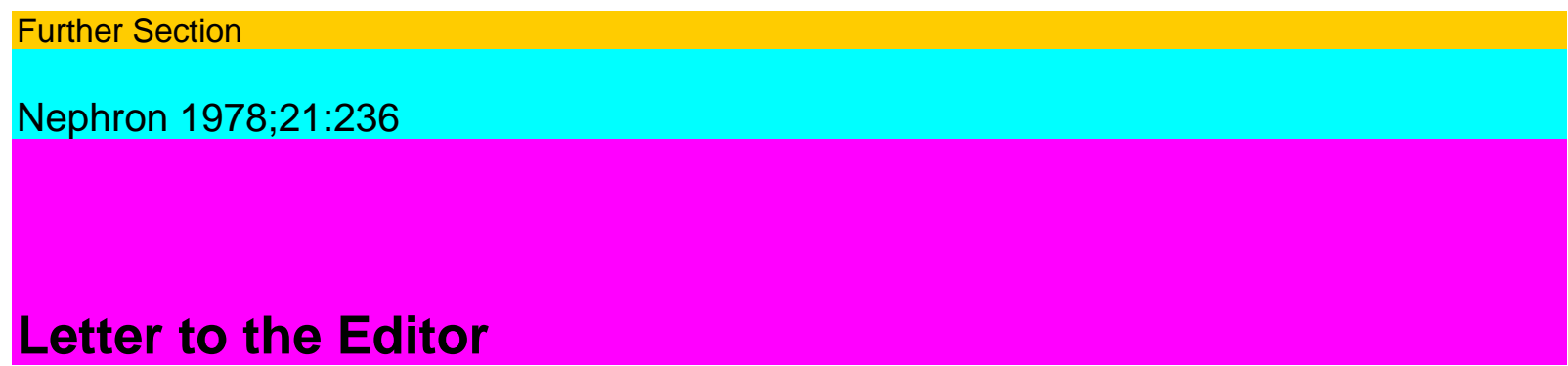

\title{
To the Editor
}

I have read with interest the article Increase in Plasma Extracellular Fluid Volume Ratio Caused by Bilateral Nephrectomy in Patients on Maintenance Hemodialysis in Nephron , vol. 20, No. 2, 1978, and would like to submit our recent experience on: Bilateral Nephrectomy and Chronic Hemodialysis in Renal Failure due to Diabetic Nephropathy Complicated by Malignant Hypertension and Cerebral Edema.

It is a well-known fact that bilateral nephrectomy will result in profound anemia [1], extracellular volume expansion [2], osteodystrophy regulation of blood pressure, and electrolyte imbalances. For this reason it is believed that bilateral nephrectomy should be reserved in critical patients who either are scheduled for renal transplantation or have uncontrolled hypertension. We have recently dealt with a diabetic male patient who underwent an unsuccessful renal transplant on January 5,1978, and was placed on maintenance hemodialysis $12 \mathrm{~h}$ weekly. His blood sugar was fairly well controlled with $40 \mathrm{U}$ of NPH insulin but his blood pressure remained around 260/160 mm Hg despite the use of combinations of methyldopa, reserpine, ßblockers, and guan-ethidine in their therapeutic ranges.

On January 27, 1978, the patient became totally blind due to papilledema and retinal hemorrhages, and on January 29, 1978, following a seizure, he became comatous. Lumbar puncture revealed that the protein and cell content of cerebral fluid were normal except that spinal fluid pressure was slightly elevated. Cerebral scan and angiogram were normal. Based on the above-mentioned findings, bilateral nephrectomy was immediately performed, and 2 days later the patient gained his consciousness, blood pressure returned to 120/70 mm Hg without the use of antihypertensives. A week later, the patient began to see and eye ground fields improved to some extent. The patient was discharged from the hospital 2 weeks later and resumed his daily activities while being on maintenance hemodialysis $18 \mathrm{~h}$ weekly.

This observation will once again confirm the importance of emergency bilateral nephrectomy in those cases whose elevated blood pressure cannot be controlled by intensive antihypertensive therapy while patients are on maintenance hemodialysis, since bilateral nephrectomy can be lifesaving in these instances.

\section{References}

Stenzel, K. H.; Cheigh, J. S.; Sullivan, J. F.; Tapia, L.; Riggio, R. R., and Rubin, A. L.: Clinical effects of bilateral nephrectomy. Am.J. Med. 58: 69-75 (1975).

Ledingham, J.M. and Pelling, D.: Hemodynamic and other studies in the renoprival hypertensive rat. J.Physiol. 270:233(1970).

Mahoney, J.F.; Gibson, G.R.; Sheil, A.G.; Storey, B.G.; Stokes, G.S., and Stewart, J.H.: Bilateral nephrectomy for malignant hypertension. Lancet/: 1036(1972).

Bijan Nik-Akhtar and Homayoon Khonsari Departments of Nephrology and Urology University of Tehran, Tehran (Iran) 